\title{
Primary Thyroid Lymphoma - A Rare Case Report
}

\author{
Sripriya Krishnan, I.V. Renuka*, C. H. Ramya, Harshitha \\ Dept of Pathology, NRI medical college and general hospital, Chinakakani, Guntur, India
}

\begin{abstract}
Primary thyroid lymphoma (PTL) is a rare condition and accounts for $5 \%$ of all thyroid malignancies. They constitute less than $2 \%$ of all lymphomas. Most PTL's are non-Hodgkin's lymphomas. It is clinically essential to discriminate between primary or secondary lymphoma of the thyroid as the treatment and prognosis varies. It commonly occurs in women in their sixth and seventh decade. Primary thyroid lymphoma is quite curable without the need for extensive surgery if diagnosed early. We present such a case of a 60 - year old female patient presenting with complaints of neck swelling with loss of appetite and dysphagia. Histopathological diagnosis confirmed Primary thyroid lymphoma of B cell origin which was confirmed by immunohistochemistry.
\end{abstract}

Keywords: Primary Thyroid Lymphoma, Hashimoto's Thyroiditis, B Cell Lymphoma, Immunohistochemistry

\section{Introduction}

Primary thyroid lymphoma (PTL) is a rare entity and is defined as a lymphoma involving only the thyroid gland or the thyroid gland and regional lymph nodes without contiguity or metastasis to other areas during the time of diagnosis. ${ }^{[1]}$ It is a rare disease and the diagnosis can be challenging and influences the treatment, as shown in this case report. Primary thyroid lymphoma is quite curable without the need for extensive surgery if recognized early. We present such a case of PTL.

\section{Case Report}

A 60 year old female patient came with previous history of swelling in front of neck for 15 years. She had complaints of further increase in size of swelling since 2 months with loss of appetite and dysphagia. She is a known case of hypothyroidism on treatment.

On examination, the swelling was approximately $7 x 5 \mathrm{~cm}$, on left side of the neck and a similar swelling was present in the right side of the neck about $3 \times 2 \mathrm{~cm}$. The swellings were firm, non tender and moving with deglutition.

$\mathrm{X}$-ray neck showed diffuse soft tissue swelling anterior and posterior to trachea at $\mathrm{C} 4$ to $\mathrm{C} 7$ levels with narrowing of trachea causing mass effect on trachea. Ultrasound revealed grossly bulky lobes of thyroid (left $>$ right) and isthmus. CECT neck showed diffuse enlargement of thyroid with no definite extrathyroid infiltration/ invasion /encasement of major vessels. No lymphadenopathy was noted.

Thyroid profile showed an elevation in TSH, T3 and T4 were normal. Other investigations were within normal limits.
Cytological Findings: FNAC was done from both right and left lobes of thyroid. The aspirate from the right lobe and left lobe showed a similar picture. The aspirate was cellular and showed a rich yield of lymphocytes, few plasma cells and some hurthle cells. The initial cytological diagnosis was Hashimoto thyroiditis, but in view of large lymphocytic predominance and occasional atypical cells, histopathological examination was advised to rule out lymphoma.

Histopathological Findings: We received a total thyroidectomy specimen, with right lobe measuring $4 \times 3.5 \times 2 \mathrm{~cm}$, left lobe measuring $7 \times 4 \times 3.5 \mathrm{cms}$ and isthmus measuring $1.5 \times 0.5 \mathrm{~cm}$. Cut section of all the lobes was solid, fleshy and grey white. Sections studied from both lobes and isthmus showed diffuse infiltration by large cells with distinct nucleoli and scant cytoplasm. There was near total obliteration of normal thyroid tissue by these cells. Most areas showed lymphoepithelial lesions with isthmus and right lobe of thyroid showing foci of hashimoto's thyroiditis (HT). So the final diagnosis of " primary thyroid lymphoma of probable B cell type " was made. IHC marker CD 20 showed diffuse positivity. The diagnosis of Primary thyroid lymphoma of B cell origin was given. The patient was referred to the medical oncologist for further treatment.

\section{Discussion}

PTL is a rare condition and accounts for $5 \%$ of all thyroid malignancies. They constitute less than $2 \%$ of all lymphomas. ${ }^{[1]}$ Most PTL's are non-Hodgkin's lymphomas. 50 to $80 \%$ of the PTL's are diffuse large B cell lymphoma (DLBCL) and, 20 to $30 \%$ are mucosa- associated lymphoid tissue (MALT) lymphomas, and majority of these are 


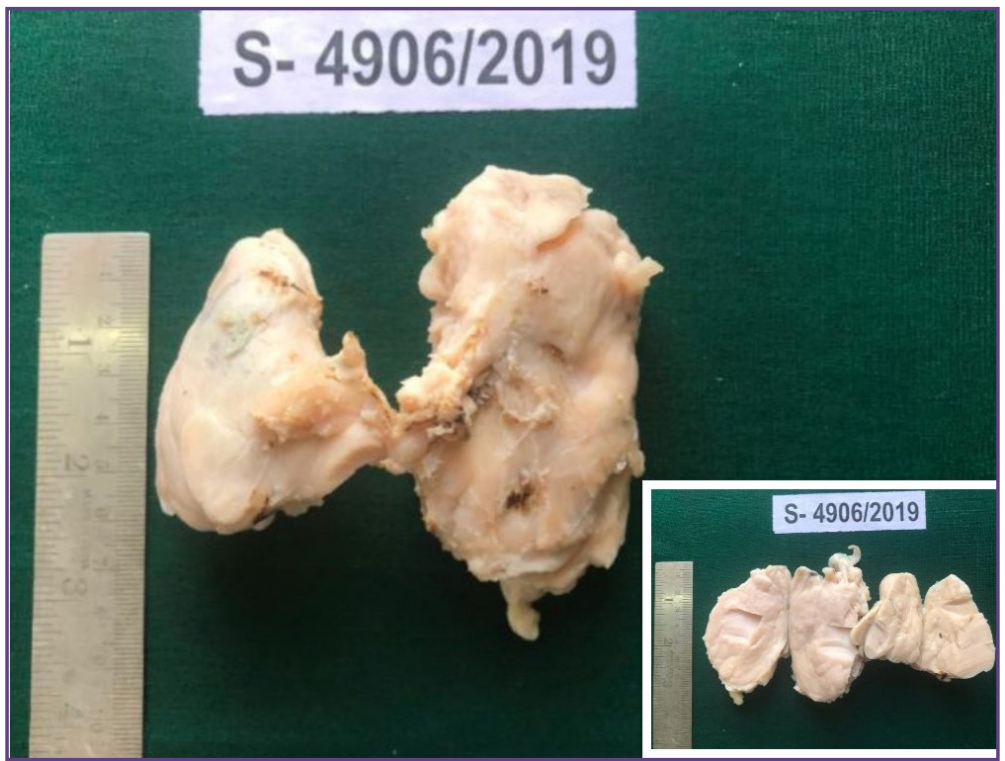

Fig. 1: Total thyroidectomy specimen, right lobe measuring $4 \times 3.5 \times 2 \mathrm{~cm}$, left lobe measuring $7 \times 4 \times 3.5 \mathrm{cms}$ and isthmus measuring $1.5 \times 0.5 \mathrm{~cm}$. Cut section of all the lobes was solid, fleshy and grey white.
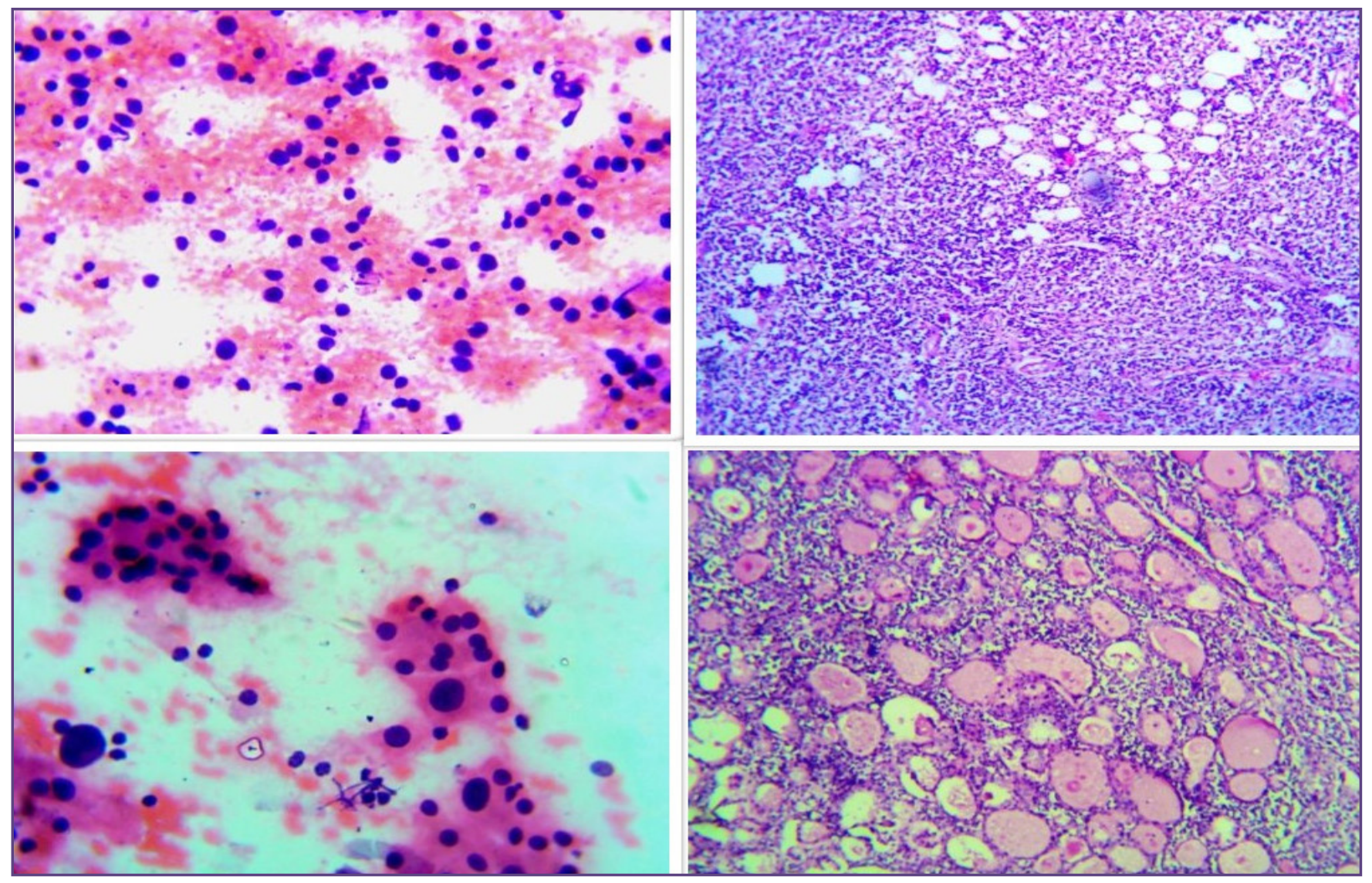

Fig. 2: A, B: FNAC( H\&E X100) showing rich lymphocytic predominance with few atypical cells giving suspicion of lymphoma and corresponding histology ( $H \&$ E X 100) showing dense infiltration by atypical lymphocytes C,D: FNAC showing an area of hashimotos thyroiditis with hurthle cells (H\&E X 400)and corresponding area showing hashimotos thyroiditis (H\&E X 100x) in tissue sections. 


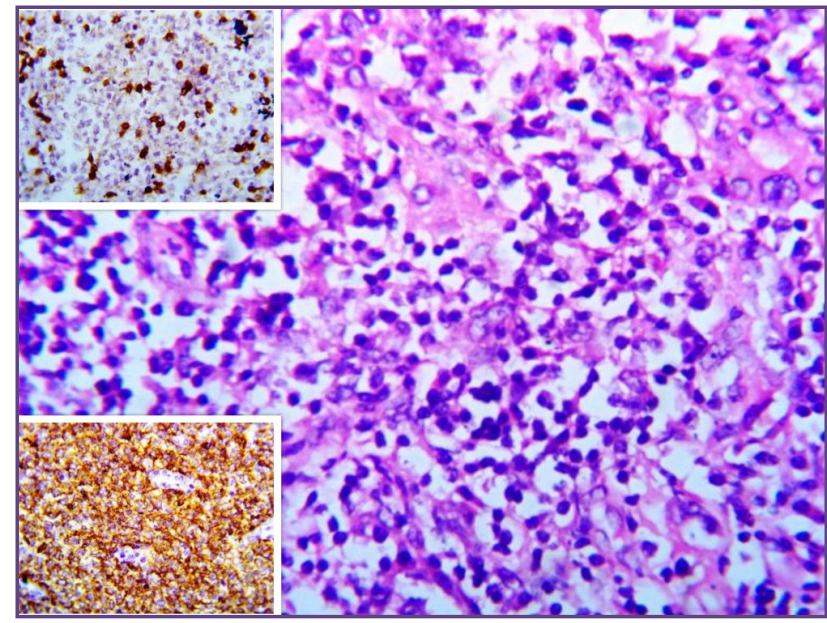

Fig. 3: Tissue section (H\&E $X$ 400) thyroid tissue showing diffuse infiltration by atypical lymphoid cells and lymphoepithelial lesions. A. CD 3 (H\&E X 400) focal positivity B. CD 20 (H\&E X 400) diffuse positivity.

extranodal marginal type. ${ }^{[2]}$ Other subtypes include follicular lymphoma (12\%), Hodgkin's disease (7\%), small lymphocytic lymphoma (4\%) and Burkitt's lymphoma $(4 \%){ }^{[2]}$

The secondary thyroid lymphoma develops from a disseminated neoplasm that metastasises to the thyroid gland. ${ }^{[2]}$ It is clinically essential to discriminate between primary or secondary lymphoma of the thyroid as the treatment and prognosis varies. The secondary lymphoma of the thyroid is a widespread disease and the mortality rate is higher, compared to primary thyroid lymphoma in early stages. ${ }^{[3]}$ PTL staging is based on Ann Arbor staging criteria. ${ }^{[4]}$

The thyroid gland generally does not contain lymphoid tissue. Under different pathological conditions, the appearance of lymphocytes may occur promoting the further development of the disease. The presence of Hashimoto's thyroiditis is a well-established risk factor, increasing the risk of developing PTL. ${ }^{[5]}$ According to a few studies, Hashimoto's disease is associated with more than $90 \%$ of the PTL. ${ }^{[6]}$ This relationship is probably due to chronic antigenic stimulation leading to malignant transformation. To clarify this argument, Moshynska et al. ${ }^{[7]}$ investigated a sequence similarity between HT and PTL. They reported clonal similarity in the IG heavy chain rearrangement sequences in the clonal bands of HT and PTL from the same patients, providing evidence that PTL may arise from HT. In our clinical case, the previous diagnosis of Hashimoto's thyroiditis was not known, but the thyroid histopathological examination proved the coexistence between the two.
Mendes et al has shown a increased risk for developing lymphoma in the setting of autoimmune disorders ${ }^{[8]}$ There seems to be a strong link between some autoimmune diseases and certain non-Hodgkin lymphoma, and thyroid MALT lymphoma in a background of Hashimoto's thyroiditis. ${ }^{[8]}$

PTL is more common in female patients (F:M ratio $3-4: 1$ ) (1), present more frequently in the sixth to seventh decade of life. ${ }^{[9]}$ As in our case, it was a 60 year old female patient. Most of the patients are diagnosed with symptoms and signs of compression of adjacent structures such as dyspnoea, dysphagia, stridor, hoarseness or coughing as our patient presented with dysphagia. ${ }^{[10]}$ Most individuals are euthyroid at presentation, but few may be hypothyroid. Our patient presented with hypothyroidism. ${ }^{[9]}$

FNAC plays a pivotal role in the diagnosis of thyroid disease. On the other hand, its role in the diagnosis of PTL is often limited because the differential diagnosis between thyroid lymphoma, lymphocytic thyroiditis, hashimoto's thyroiditis and even anaplastic carcinoma thyroid is very difficult in some cases, presenting a real challenge. As seen in our case, the cytological diagnosis was hashimotos thyroiditis, but the lymphocytic predominance raised a suspicion of lymphoma.

The therapeutic approach in PTL is controversial because of its rarity. ${ }^{[6]}$ The control of the disease is achieved by radiotherapy, surgery or both. Chemotherapy is used for control of disseminated disease, to improve long term outcome. ${ }^{[6]}$ The surgical treatment in PTL is questionable.

In this case report, the patient underwent a total thyroidectomy as she presented with compressive symptoms. Post operative period was uneventful and the patient was discharged. She was referred to the medical oncology department for further treatment.

\section{Conclusion}

To conclude, it is important to consider the diagnosis of primary lymphoma of thyroid in patients presenting with enlarging neck mass and with a previous history of Hashimotos thyroiditis.

\section{Acknowledgements}

Nil

\section{Funding}

Nil

\section{Competing Interests Nil}

Ethical clearance was obtained from the institute's ethics committee for reporting this case. 


\section{References}

1. Chan, J.K.C, Burke J.S, Ferry J.A, Wotherspoon A., Hematolymphoid tumours. In: et al. (eds.) WHO Classification of Tumours of Endocrine Organs. Lyon: IARC; 2017:137-8.

2. Chen $\mathrm{C}$ et al., Primary thyroid T-lymphoblastic lymphoma: a case report and review of the literature, Int. J. Clin. Exp. Pathol.2014;7 (1):443-450.

3. Takashima S, et al., Secondary malignant lymphoma which simulated primary thyroid cancer, J. Clin. Imaging.2000;24:162-165.

4. Graff-Baker, J.A. Sosa, Roman S.A, Primary thyroid lymphoma: a review of recent developments in diagnosis and histology-driven treatment, Curr. Opin.Oncol. 2010;22:17-22.

5. Sakorafas G.H., Kokkoris P, Farley D.R., Primary thyroid lymphoma - diagnostic and therapeutic dilemmas, Surg. Oncol.2010;19:e124-e129.

6. Widder S, Pasieka J.L, Primary thyroid lymphomas, Curr. Treat. Options Oncol. 2004;5 (4) :307-313.

7. Mo shynska OV, Saxena A. Clonal relationship between Hashimoto thyroiditis and thyroid lymphoma. J Clin Pathol 2008;61: 438-444

8. Teixeira Mendes L.S, Wotherspoon A, Marginal zone lymphoma: associated autoimmunity and auto-immune disorders, Best Pract. Res. Clin. Haematol.2016;1-12

9. Katna R, Shet T, Sengar M, et al., Clinicopathologic study and outcome analysis of thyroid lymphomas: experience from a tertiary cancer center,Head Neck 2013; 35:165-171.

10. Derringer G.A, Thompson L.D, Frommelt R.A al., Malignant lymphoma of the thyroid gland: a clinicopathologic study of 108 cases, Am. J. Surg. Pathol.2000;24:623-639.

*Corresponding author:

Dr. I.V. Renuka, Professor, Dept of pathology, NRI medical college and general hospital, Chinakakani, Guntur, India

Email: repriya56@gmail.com

Date of Submission : 27/11/2019

Date of Acceptance : 13/04/2020

Financial or other Competing Interests: None.

Date of Publication : 27/06/2020 